\title{
Investigation into the control strategy for a long spine of Edinburgh Duck modules, using an efficient numerical model
}

\author{
Alfred Cotten and David I. M. Forehand
}

\begin{abstract}
An efficient numerical model of a spine of ten Edinburgh duck modules is developed. The spine joints and duck modules are modelled using a linear approach based on the theory of generalized modes, which mitigates the need for a more computationally expensive timedomain solver. This approach also allows for computation of the shear forces acting on the spine joints, and has the added benefit of enabling the use of complex conjugate control. The resulting hydrodynamic model is verified for a three duck spine against an alternative implementation that uses a nonlinear multibody solver to enforce the joint motions. A conservative weighted motion constraint is imposed on the controlled degrees of freedom of the ten duck spine, in order to ensure results stay within the bounds of the linear theory. Pertinent sections of the theory underpinning the constrained complex conjugate control method are elaborated upon for the case in which not all degrees of freedom are controlled. An implementation of this control method for a solo duck is compared against a result from the literature, in order to confirm the suitability of the choice of duck design in this study. The control force coefficients that maximise the absorbed power, subject to the motion constraint, are computed for the ten duck spine over a range of wave periods and wave heading angles. The resulting dynamics of the spine of ducks are explored, with particular emphasis on aspects related to the power extraction and forces acting within the system.
\end{abstract}

Keywords-Complex conjugate control, Edinburgh duck, generalized modes, numerical modelling, wave energy converter.

\section{INTRODUCTION}

W HEN first proposed as a concept to extract large amounts of energy from sea or ocean waves, long spines comprising many tens of 'duck' devices were envisioned, in order to take advantage of the common reference structure [1]. Since then, many stages of

Manuscript received 16 March; accepted 27 March; published 30 November, 2020. This is an open access article distributed under the terms of the Creative Commons Attribution 4.0 licence (CC BY https://creativecommons.org /licenses/by/4.0/). This article has been subject to single-blind peer review by a minimum of two reviewers. This work was supported by a studentship provided by the EPSRC through the Wind and Marine Energy Systems Centre for Doctoral Training, under award reference 1809924. A. Cotten and D. I. M. Forehand are with the Institute for Energy Systems, School of Engineering, University of Edinburgh, Edinburgh, EH9 3JL, Scotland, UK (emails: Alfred.Cotten@ed.ac.uk, D.Forehand@ed.ac.uk). Digital Object Identifier https://doi.org/10.36688/imej.3.145-154 investigation have led to multiple design refinements. With a view to deep-water deployment, the flexibility afforded by rotational joints is necessary between the ducks to avoid significant damage by bending moments [2]. Given the subsequent lack of rigidity of the spine, effective designs for a power take-off system were required to be housed inside each duck. These were initially provided by use of a gyroscopic reference frame [3], and more recently by hydraulic technology. Presently, work on the design of full-scale hardware with personnel access to all power-conversion components, spine section-replacement in moderate sea states and dry power collection to $250 \mathrm{MW}$ is ongoing. These designs, crucially, retain the absence of a rigid reference point, and enable power conversion that can be continuously controlled. It is also proposed for the hydraulic technology to allow power extraction through the joints, giving full control over most of the degrees of freedom associated with a full spine of ducks. It is this type of integrated, freely-floating system that is required to enable truly significant levels of wave energy extraction from our seas and oceans. Perhaps more importantly, this kind of spine-based system is able to maximally exploit the available sea or ocean space, given the demands of other users. Designated shipping lanes perforating a wellmarked spine of ducks also provide advantages for ship navigation.

In addition to the efficient geometric design, maximal power extraction necessitates active control of the system during operation. To this end, a pitch-heave-surge rig was used in a narrow wave tank to mimic the behaviour of a single member of a full spine of ducks [4]. An automated machine learning technique was used to tune seven control force coefficients for maximal power extraction in a selection of irregular sea states. Power could be extracted through all three degrees of freedom, akin to the manner in which power would be extracted by a member of a full spine via the flexure of the joints and the pitching of the ducks. A key finding was that the optimisation process often led to increased proportions of the total power deriving from the heave and surge motions, especially in longer period waves. This suggests that significant power extraction from the joints of a full spine can be expected. Related work on long spines of cylindrical modules, not optimised for power extraction, found that oblique wave directions led to much larger 
bending moments at the down-wave end of the spine [5]. It was believed that flexural waves travelling along the spine were a precursor to this phenomenon, and only occurred with specific heading angles of the waves. It was also suggested that these features could perhaps be exploited by the control moments to yield increased power extraction, such as was achieved with Pelamis [15]. The complexities encountered in these two studies shed some light on the expected behaviour, but it is not clear how these phenomena would manifest in a full spine of ducks optimised for power extraction.

This work presented herein aims to make some progress towards bridging the large gap between the two aforementioned studies. An efficient numerical model of a spine of ten Edinburgh ducks is used to explore the device dynamics under operating conditions that simulate optimal power extraction. Since the numerical model is based upon linear hydrodynamics and assumes small body motions, the control force coefficients are optimised under motion constraints, in order to prevent unrealistic excursions from the water surface. To this end, a semi-analytical approach is adapted to account for the fact that not all degrees of freedom of the freely-floating spine of ducks are controlled. The model is then used to investigate several aspects relating to the system dynamics, including the power generation and shear forces acting on the joints.

\section{NUMERICAL MODEL}

The duck design used in this study is based on the cross-sectional shape of the D0018 Medium Beak Duck from [6], which was designed to be similar to a member of a full spine. The six ballast tubes from that design are augmented here by a seventh, located much lower in the duck body to help achieve the desired mass distribution (Fig. 1).

The ballast tubes are each of constant density and are $1.27 \mathrm{~m}$ in diameter. Their masses must be selected such that a suitable waterline height and an appropriate centre of gravity location are achieved, whilst satisfying the constraint that the centre of gravity and centre of buoyancy both lie on the same vertical line. The masses used (Table I) result in a waterline height of $4.12 \mathrm{~m}$ above the centre of rotation, and a centre of gravity $1.65 \mathrm{~m}$ radially outwards from the centre of rotation, at an angle of $4^{\circ}$ anticlockwise from the centreline. This angle is

TABLE I

BALLAST TUBE MASSES AND POSITIONS

\begin{tabular}{cccc}
\hline $\begin{array}{c}\text { Ballast } \\
\text { index }\end{array}$ & Mass $(\mathrm{kg})$ & $\begin{array}{c}\text { Radial } \\
\text { position }(\mathrm{m})\end{array}$ & $\begin{array}{c}\text { Angular } \\
\text { Position }\left(^{\circ}\right)\end{array}$ \\
\hline 1 & 0 & 10 & 0 \\
2 & 456000 & 5.5 & 45 \\
3 & 312000 & 4.75 & 65 \\
4 & 134000 & 6 & 0 \\
5 & 0 & 4.75 & -65 \\
6 & 56000 & 5.5 & -45 \\
7 & 752500 & 4 & -120 \\
\hline
\end{tabular}

much smaller than that used in [7], but is closer to the value of around $10^{\circ}$ recommended for good capsize recovery [8]. The ballasts are fully defined in Table I, with the radial positions defined from the centre of rotation, and the angular positions defined clockwise from the part of the centreline joining the centre of rotation and the beak tip (the pointed edge of the duck).

The centreline is orientated at $36^{\circ}$ to the horizontal (as used in [7] for a solo duck), and the distance from the centre of rotation to the beak tip along the centreline is $11.8 \mathrm{~m}$. A width of $29.5 \mathrm{~m}$ is used for each duck, with a $10.5 \mathrm{~m}$ spacing between each pair of adjacent ducks, similar to early models [5],[8]. The individual duck used here is statically stable in both pitch and roll, ensuring that the whole device is statically stable. The spine consists of ten ducks, which is deemed the minimum number to achieve adequate stability by spanning multiple wave crests [2].

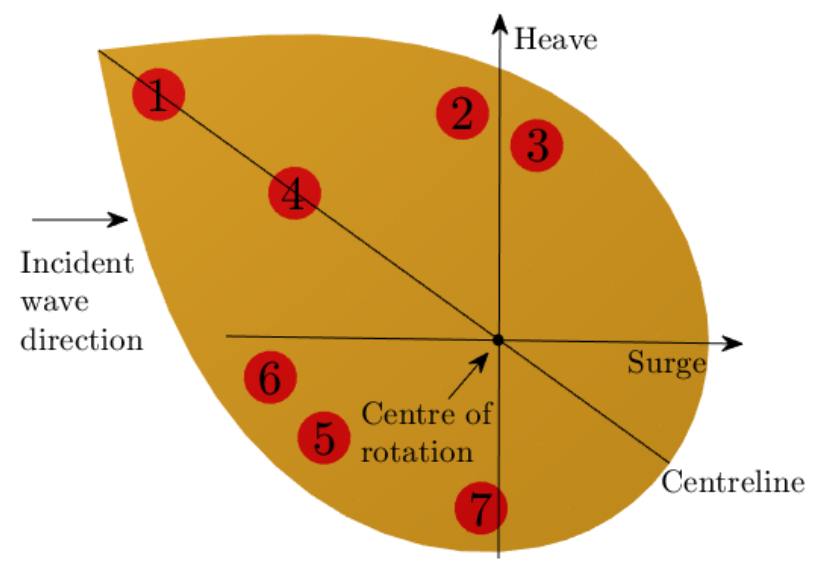

Fig. 1. Cross-section of a single member of the full spine of ducks, with the seven ballast tubes displayed. Sway is defined into the page, with the axes centred halfway along the spine length.

The hydrodynamic model of the full spine is created using the commercial radiation/diffraction code, WAMIT [11]. Linear hydrodynamic coefficients are computed, which enable the subsequent analysis of motions and forces due to incident monochromatic waves. To visualise the device, first consider a rigid spine with 5 degrees of freedom - surge, sway, heave, roll and yaw, but not pitch. Adding to this spine 10 ducks, each of which can undergo pitch rotation, and 9 joints, each of which enables rotation in the roll and yaw directions, results in a 33 degree of freedom system $(5+10+(9 \times 2))$. In order to represent these 33 degrees of freedom in the hydrodynamic model, generalized modes of motion are used (see e.g. [12]), which minimises computational demand by working entirely within the frequency-domain. Generalized modes allow the specification of extra types of motion of one part of the body, relative to the standard 6 rigid body modes (surge, sway, heave, roll, pitch and yaw). The extra velocity potentials associated with these new types of motion can then be solved for, and system motions and 
forces analysed in the exact same manner as with the rigid body modes. Given the nature of the power take-off systems used in this application, each of the ten ducks can be modelled as if able to react against a rigid reference frame in the pitch direction. Hence it is most convenient to define a generalized mode describing a rotation about the centre of rotation for each duck, whilst locking ('fixing') the rigid body pitch mode. This ensures that pitch motions of each duck are defined relative to a stationary reference frame, so that any applied damping or stiffness forces are applied as they would be by an internal power take-off. Conversely, any forces applied about a spine joint must involve equal and opposite reaction forces on the ducks either side. The most convenient way to do this is to define the generalized modes relative to the rigid body yaw and roll modes, which define the motions of the entire freely-floating assembly. This permits the reaction forces to be provided through the inertial coupling between the generalized modes of the joints, whilst preserving the freely-floating nature of the whole assembly of modules. These 28 generalized modes describe all of the motions through which power can be extracted, whilst the 5 rigid body modes (defined over the entire assembly of modules) are uncontrolled, as a consequence of the freely-floating nature of the whole spine.

In this study, the shear forces at the joints are also of interest. These can be analysed by defining further generalized modes, which are then specified as 'fixed', just as with the rigid body pitch mode. This equates to solving for all of the velocity potentials and enabling the hydrodynamic forces to be evaluated for all modes. However, the fixed modes are excluded from the equations of motion. The total shear forces in each defined direction can then be obtained by summing the hydrodynamic and inertial forces. Three additional generalized modes are defined for shear forces at each joint in each axial direction, giving a total of 60 modes ( 33 $+(9 \times 3))$ for which the velocity potentials are solved.

In order for the body motions and forces to be correctly defined, both the inertial and gravitational couplings between each pair of modes needs to be defined. Parametric definitions of the curves describing the duck edges are required for this due to the non-orthogonal coordinate system used to define the duck shape given in [6]. The hydrodynamic and inertial properties of the connections between ducks are neglected, and so the inertial properties and discretised mesh (Fig. 2) for the whole spine only need to take into account the shape of the ducks and the spacings between them.

Since this method of modelling involves manual entry of the inertial and gravitational properties of each of the generalized modes of motion, further verification of the modelling approach has been carried out using an alternative modelling approach. The alternative approach computes hydrodynamic coefficients for the individual duck bodies as if they were mechanically disconnected,

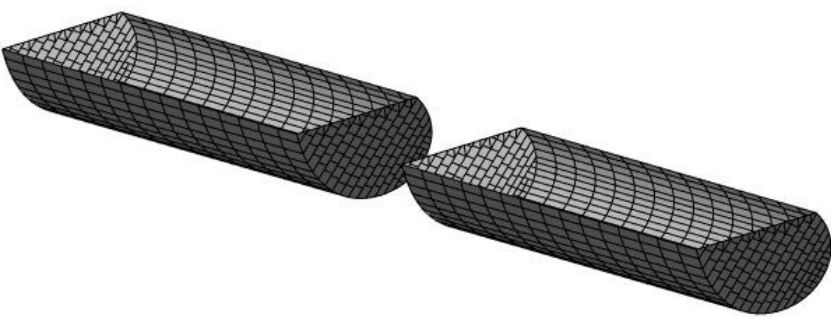

Fig. 2. A section of the discretised mesh used to compute the hydrodynamic forces that act on the submerged part of the ten duck spine.

but hydrodynamically interacting. The joints are then implemented by imposing constraint equations on the system using a multibody package. Since the constraint equations are nonlinear, they must be solved in the timedomain. Much greater computational expense is required for this approach, especially given the need for more radiation potentials than there are degrees of freedom in the spine. The system can then be solved for the body motions, using small incident wave amplitudes to ensure dynamics are within the linear regime. Given enough settling time to reach a steady state, the Response Amplitude Operators (RAOs) can then be extracted from the time series. Figs. $3 \& 4$ shows a comparison between the amplitudes of the RAOs obtained from both methods, for an undamped spine of three ducks, with an incident wave heading of $30^{\circ}$. A lack of runtime of the timedomain model explains the discrepancy for higher wave periods. In the case of the joint RAOs, the discrepancy at lower wave periods could be explained by the numerical differences in modelling the two systems, especially given the lower absolute values.

TABLE II

SUMMARY OF MODEL MODES (RIGID BODY + GENERALIZED)

\begin{tabular}{|c|c|c|c|}
\hline $\begin{array}{l}\text { Mode type, } \\
\text { free }\end{array}$ & Quantity & $\begin{array}{l}\text { Mode type, } \\
\text { fixed }\end{array}$ & Quantity \\
\hline Rigid body & 5 & $\begin{array}{l}\text { Joint shear } \\
\text { forces, surge }\end{array}$ & 9 \\
\hline Duck pitch & 10 & $\begin{array}{l}\text { Joint shear } \\
\text { forces, sway }\end{array}$ & 9 \\
\hline $\begin{array}{l}\text { Joint flexure } \\
\text { (in roll \& yaw } \\
\text { directions) }\end{array}$ & 18 & $\begin{array}{l}\text { Joint shear } \\
\text { forces, heave }\end{array}$ & 9 \\
\hline Total: & 33 & Total: & 27 \\
\hline
\end{tabular}

\section{CONTROL StRATEgY}

An additional benefit of formulating the model entirely within the linear boundary value problem that describes the interaction of the duck spine with linear waves, is that complex conjugate control [14] is applicable. It is known that the standard form of complex conjugate control can demand unrealistically large motions at higher wave periods. One way to prevent this involves applying a single, weighted constraint to the body motions [9]. In this application, only the damping and stiffness coefficients of the 28 controlled degrees of freedom should be optimised, in order to give maximal power extraction subject to the weighted constraint. 

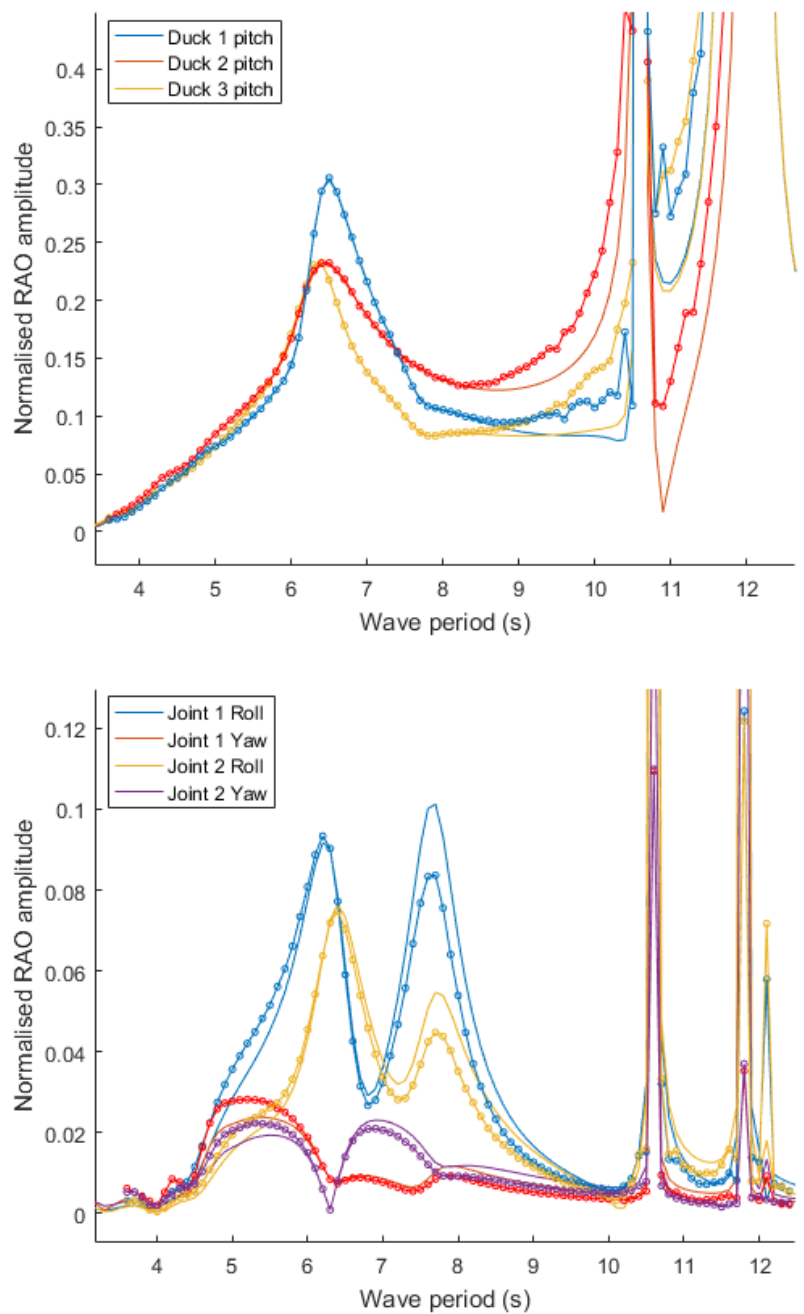

Figs. 3 (upper) and 4 (lower). Verification of the modelling approach. Solid lines with circles: time-domain approach. Solid lines: frequency-domain approach using generalized modes.

The approach for applying complex conjugate control methods to only a subset of the total number of modes of motion, is briefly discussed in [10], but we expand upon that here, bearing in mind the application to the duck spine.

Through coupling terms in the equations of motion, each mode of motion is dependent on each other mode of motion. This enables the equations of motion for the controlled degrees of freedom to be expressed in terms of the velocities of only those controlled degrees of freedom (see eq. (1)). The dependence of the controlled velocities on the uncontrolled velocities is instead captured by modified forms of the impedance matrix and the vector of excitation forces. (See equation 32 in [10] for more explanation.) Let $F_{c}$ denote the vector of control forces/moments, $Z_{c c}^{m}$ the modified impedance matrix (again, see [10]), $U_{c}$ the vector of controlled velocities, $A$ the wave amplitude, and $X_{c}^{m}$ the modified excitation force vector. Then

$$
F_{c}=Z_{c c}^{m} U_{c}-A X_{c}^{m}
$$

Expressing the power in terms of these control forces, leads to (2), where $*$ denotes the complex conjugate, and $B_{c c}$ is the real part of the modified impedance matrix.

$$
\begin{aligned}
& P=\frac{A^{2}}{8} X_{c}^{m *} B_{c c}^{-1} X_{c}^{m} \\
& -\frac{1}{2}\left(U_{c}-\frac{A}{2} B_{c c}^{-1} X_{c}^{m}\right)^{*} B_{c c}\left(U_{c}-\frac{A}{2} B_{c c}^{-1} X_{c}^{m}\right)
\end{aligned}
$$

Then by applying a similar approach based on the method of Lagrange multipliers to that given in [13], the optimal velocities can be obtained, and used in combination with (1) and the fact that the control forces are proportional to the velocities, to obtain the control matrix.

$$
C=Z_{c c}^{m^{*}}+2 \mu \Gamma_{c}^{-2}
$$

where $\mu$ denotes the Lagrange multiplier, and $\Gamma_{c}$ is the matrix of velocity constraint weightings.

Similar treatment for the unconstrained case yields that control matrix.

$$
C=Z_{c c}^{m^{*}}
$$

For each pairing of wave period and heading angle, if the wave amplitude is large enough for the motion constraint to restrict the power, the Lagrange multipliers are numerically computed, based on the theory given in [9]. Otherwise, regular complex conjugate control is employed. Hence, depending on the wave amplitude, wave period and wave heading angle, (3) or (4) is used to determine the power take-off stiffness and damping coefficients for the 28 controlled modes. These coefficients can then be combined with the hydrodynamic model to obtain the motions and control forces associated with all 33 'free' modes, plus the shear forces acting through the 27 fixed modes. The power extracted in regular waves of frequency, $\omega$, can then be computed using (5). $B_{\text {РтO }}$ is the real part of control matrix $C$, and $\xi$ are the motions corresponding to velocities $U_{c}$.

$$
P=\frac{1}{2} \omega^{2} \xi^{*} B_{P T O} \xi
$$

For further verification that the control method is implemented correctly, the power was computed for a solo duck in waves of $1 \mathrm{~m}$ amplitude and a $40^{\circ}$ heading, and compared to the equivalent result from [9] (Fig. 5). The plots are very similar, despite a slightly broader plot for the duck used in the present study. Maximal performance differs a little due to the different geometries and mass distributions, but is deemed similar enough to substantiate the use of the duck design introduced in this study. 


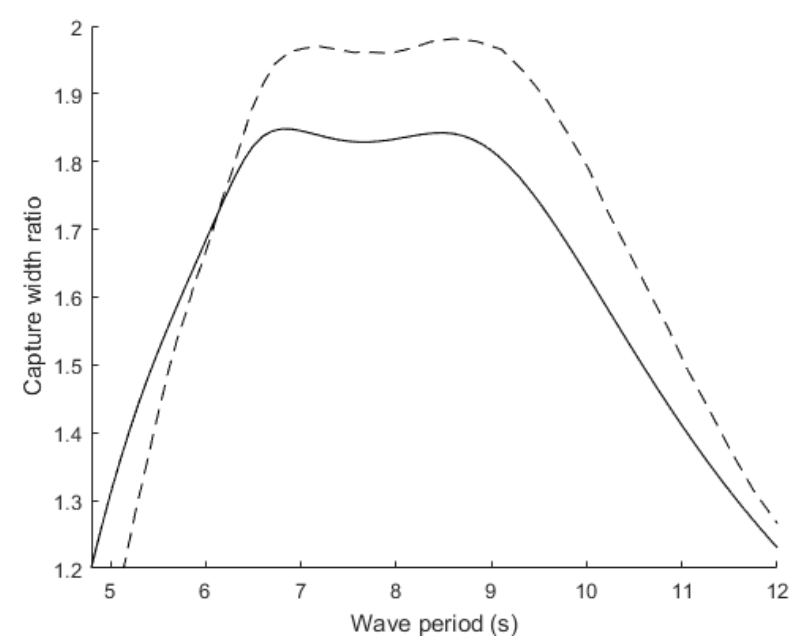

Fig. 5. Efficiency of single duck in $40^{\circ}$ oblique waves, $1 \mathrm{~m}$ wave amplitude, with motion constraints. Dotted line: Result from [9]. Solid line: Result from the present study.

\section{RESUlTS AND DisCUSSION}

The hydrodynamic model of the spine of ducks was used in conjunction with the constrained form of complex conjugate control over a range of wave periods (4 to $15 \mathrm{~s}$ in $0.1 \mathrm{~s}$ increments) and incident wave angles (0 to $90^{\circ}$ in $10^{\circ}$ increments). $0^{\circ}$ corresponds to the case where the incident wave direction is perpendicular to the spine, and at $90^{\circ}$ the wave direction is parallel to the spine. Though the range of wave periods likely to be encountered in a real sea may go beyond the upper limit of $15 \mathrm{~s}$, this is deemed large enough to gain useful insight into the behaviour of a full duck spine in this study. The weighted global constraint was set following the example in [9], using motion limit weightings of $0.5 \mathrm{rad}$ for the pitch angle of each duck and 0.2rad for each joint degree of freedom. These provide a particularly conservative case in this application, given that a larger number of degrees of freedom increases the severity of the constraint, when using the same constraint weightings. The wave amplitude was set to $1 \mathrm{~m}$ for all wave conditions.

Devising a fair measure of performance for a device based on multiple operating principles (i.e. extracting power through different types of degrees of freedom) is not trivial. Capture Width Ratio (CWR) provides insight into the efficiency of a device, but the spine of ducks can extract power through acting both as a terminator (ducks pitching) and as an attenuator (joints flexing). Both these modes of operation can readily occur simultaneously, especially in oblique waves. Considering its operation as a terminator device, when the primary power generation will be due to the ducks pitching, it is also not clear what to set as the characteristic length for the CWR. The duck spine is somewhat like a tightly-packed, one-unit-deep array of devices, albeit with jointed connections to stabilise their locations relative to one another. The question remains whether it is fairer to use the entire spine length or just the width spanned by the ducks themselves. It is also unclear as to whether CWR can fairly be used to compare the inherent value of the duck pitch motions with that of the joint flex motions. Despite these doubts, CWR can give a useful indication of performance. The total length of the spine is adopted here as a conservative characteristic length, for all modes of power generation, but it should be borne in mind that it is not appropriate to compare the CWR used in this paper to CWRs of other devices. The metric is simply used to give a better indicator of performance in sea states of different energies, than power alone would. Fig. 6 shows good performance over a wide range of wave periods and angles. The highest efficiencies can be maintained for a wide range of wave periods, especially if the orientation of the spine can adapt sufficiently as the predominant wave period changes. The results here suggest that a duck spine may yield optimal performance when angled away from the incoming wave direction by somewhere between $10^{\circ}$ and $30^{\circ}$. However, enlarging the ducks could mitigate any further required increase in the orientation angle of the spine. A zigzag spine configuration may also be able to achieve the higher performance, whilst avoiding the need to skew the overall orientation of the spine. Additionally, the motion constraints impact the power extraction most heavily for the lowest wave angles, particularly $0^{\circ}$ and $10^{\circ}$. Since these are the regions in which more power comes from the duck pitch motions, it is clear that the overly restrictive constraints bias against the duck pitch power extraction. Whilst it was not in the scope of the present study to assess the full power generating potential of the duck spine, future work should investigate the appropriateness of the motion constraints, and indeed duck shape and design.

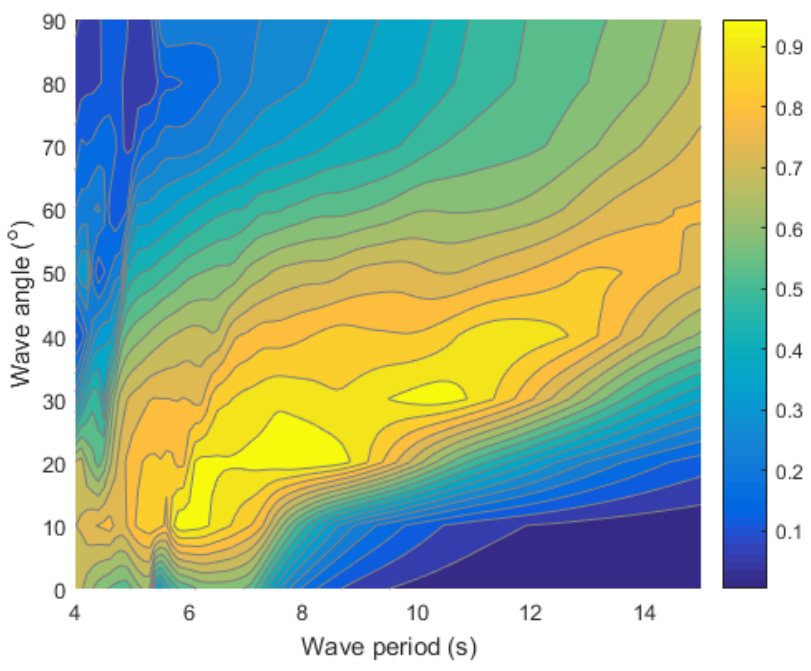

Fig. 6. Total capture width ratio of the duck spine system under constrained optimal power generation.

This performance profile is provided by the complementary power extraction through the three types of rotation (duck pitch, and roll and yaw about each joint). In general, the ducks provide more power than the joints at lower wave periods, even for the most oblique of wave angles (Fig. 7). Beyond a wave period of around 8s, the ducks produce a much diminished amount of power, 
even for $0^{\circ}$ waves (Fig. 8). This suggests that the scale of the ducks chosen here would be too small for many sea states, in which more energy is contained in wave components of period greater than 8s. One must also bear in mind the previously noted point on the stringency of the motion constraints.

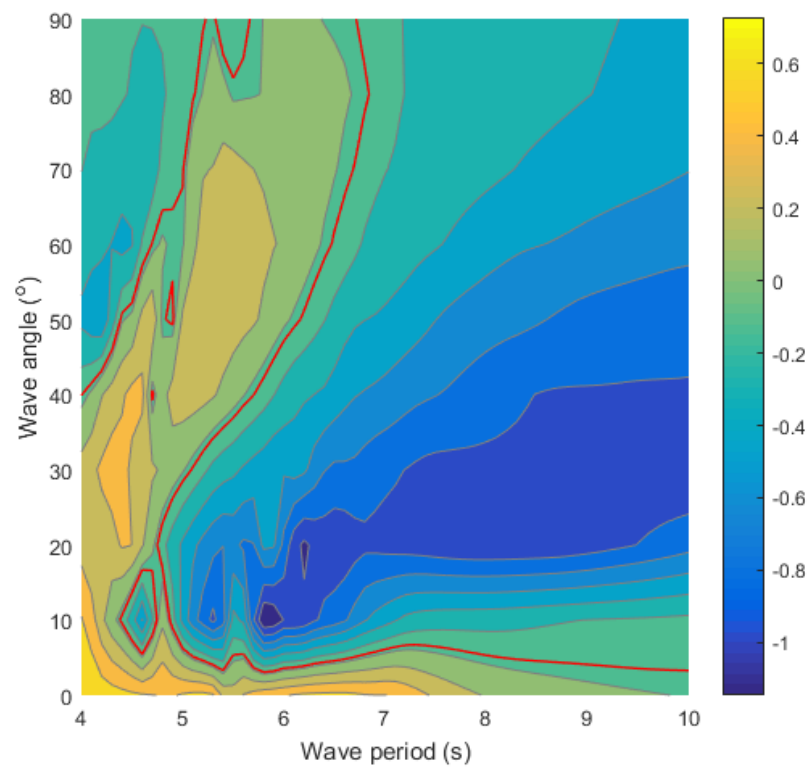

Fig. 7. Difference in capture width ratio contributions from duck pitching and joint flexing. In yellow regions, more power is extracted through pitching of the ducks than by the flexing of the joints. The red line shows the boundary along which capture width contribution of duck pitching and joint flexing is equal.

Whilst the peak in power extracted through the ducks pitching is achieved with $0^{\circ}$ incident waves (Fig. 8), the joints tend to require obliquity of the incoming waves for significant power extraction (Figs. 9, 10). Through the yawing of the modules about the joints, the most significant amounts of extracted power occur for longer wave periods and heading angles around $45^{\circ}$ (Fig. 10). As the wave period becomes much longer and as the heading angle approaches $90^{\circ}$, the amount of power extracted through the roll motions about the joints increases (Fig. 9). In the upper right corner of Fig. 9, very large amounts of power are generated, with the duck spine more optimally configured to operate as an attenuator device, e.g. Pelamis [15]. However, the efficiency is lower in these higher wave periods (Fig. 6), and so the device is better operated in a region where the ducks are designed to pitch more significantly.

Under the current control strategy, net power is often input to various degrees of freedom, in order to increase the net power extraction through other degrees of freedom. This can even be seen over groups of degrees of freedom. At a wave angle of $10^{\circ}$ and wave periods below $6 \mathrm{~s}$, the power extracted by the joint flexure in roll is particularly high (Fig. 9). Those three peaks in power extraction are mirrored by peaks of net power input (i.e. negative power extraction) through the duck pitch and joint yaw degrees of freedom. In other words, the high amount of power extracted at low wave periods through the joint roll modes must be accompanied by large power input into the joint yaw and duck pitch modes.

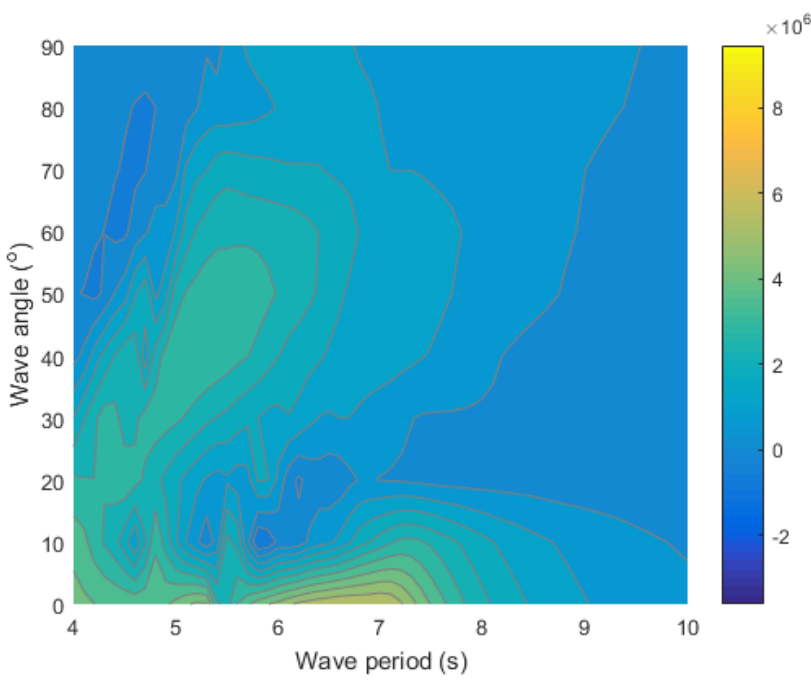

Fig. 8. Power extraction through duck pitch motions, measured in Watts.

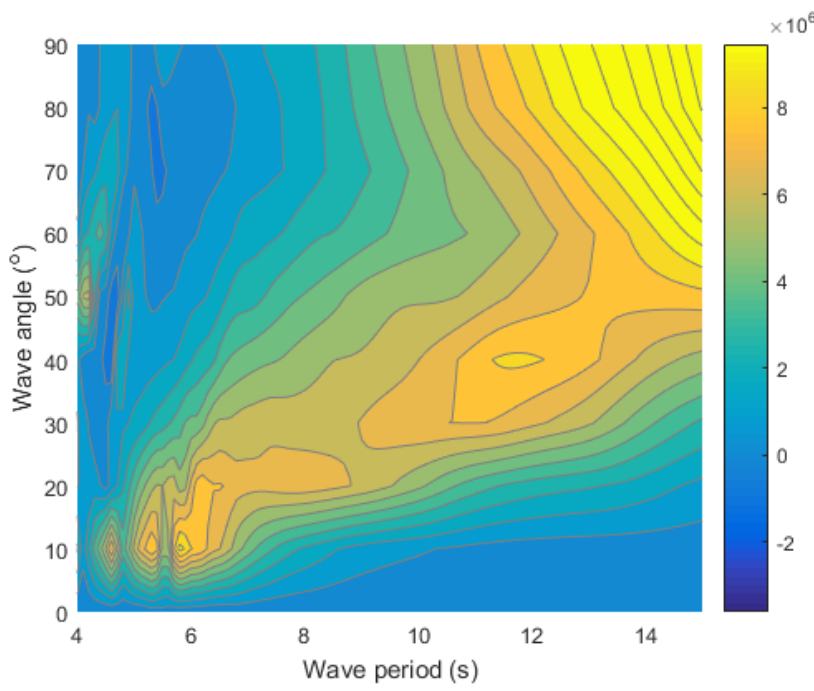

Fig. 9. Power extraction through joint roll motions, measured in Watts.

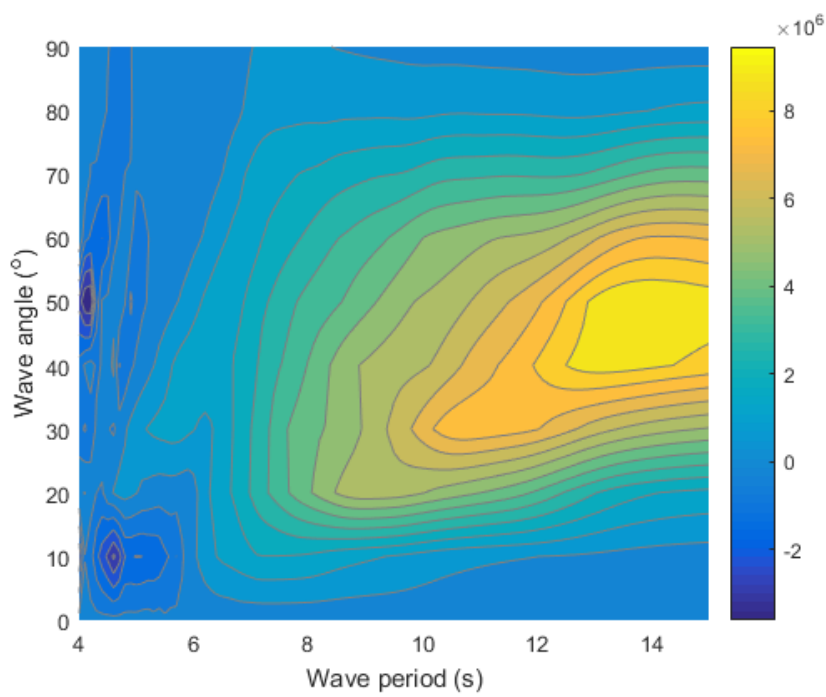

Fig. 10. Power extraction through joint yaw motions, measured in Watts.

Hence, one cannot make any inferences about the relative importance of the various degrees of freedom on the basis 
of the breakdown of the power extraction alone; higher powers extracted through one type of degree of freedom may only be possible in the presence of strong coupling with other degrees of freedom. Nonetheless, it is evident that significant amounts of power can be derived from the spine joints, even when considering just small incident wave angles, which may be expected in the directional spread of a full sea state.

As a result of the control strategy, some interesting trends emerge regarding the relative amount of power extraction via different degrees of freedom. The power extraction of joints closer to the centre of the spine is often more dominant in different types of sea state to the power extraction by joints toward the ends of the spine (Fig. 11 shows an example of this). A positive difference in power corresponds to more power being extracted through the end joints than central ones. A series of bands are present in the example of Fig. 11, corresponding to complementary behaviour of the outermost and innermost spine joints. With a $3 \mathrm{D}$ view, this is seen as a series of waves in the period-angle coordinate space. This behaviour is accompanied by much higher peak power extraction through the innermost joint roll modes, demonstrated by the blue and green regions of Fig. 11 (also note the difference in scale for the extremes in colour). As shown by the complementary orange and yellow regions, however, the power extracted via the outermost joint roll modes is much lower, but more consistent across all sea states. The high difference in these regions is thus mainly caused by greater power input to the innermost joint roll modes, for the benefit of greater power extraction elsewhere.

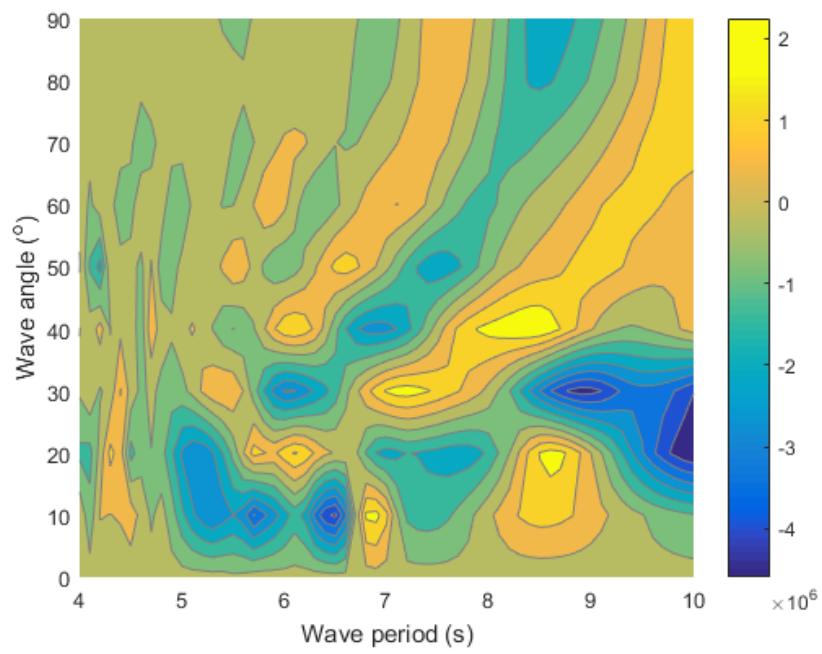

Fig. 11. Difference in power extraction through roll degrees of freedom of joint 9 (aft joint) and joint 5 (central joint); Power (joint 9 roll) - Power (joint 5 roll). Measured in Watts. Note the difference in the sizes of the positive and negative extreme values.

Trends along the spine in the power extraction from duck pitch modes are more elusive, with the behaviour appearing to contain more complexity. Peak power extraction by ducks closer to the downstream end of the spine occurs at an angle of $0^{\circ}$, whilst for ducks at the upstream end, this occurs at $10^{\circ}$ (e.g. Fig. 12). For most ducks, there are sea states in which they are net power extractors, and others in which they are net power injectors. Often, this sink-source type of behaviour occurs in closely separated regions of the period-angle space (e.g. Fig. 12).

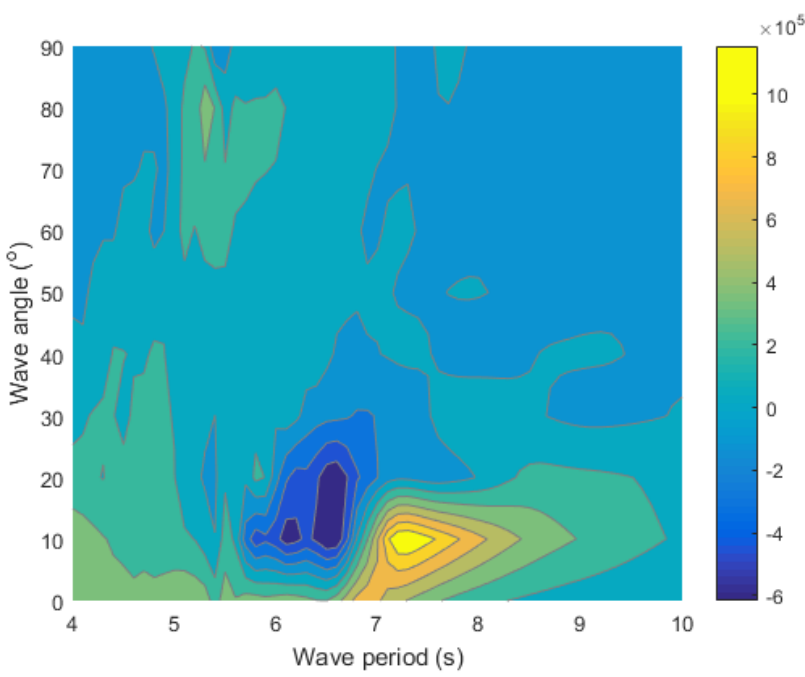

Fig. 12. Power extraction via pitching of duck 1 (upstream end of spine), measured in Watts.

Also of interest are the shear forces and control moments involved in the power-extracting duck spine. For joint roll degrees of freedom and small wave angles $\left(0^{\circ}\right.$ to $\left.20^{\circ}\right)$, the moments required by the control strategy tend to be greater towards the centre of the spine (Fig. 13). Similar trends hold for the control moments imparted at the joints in yaw.

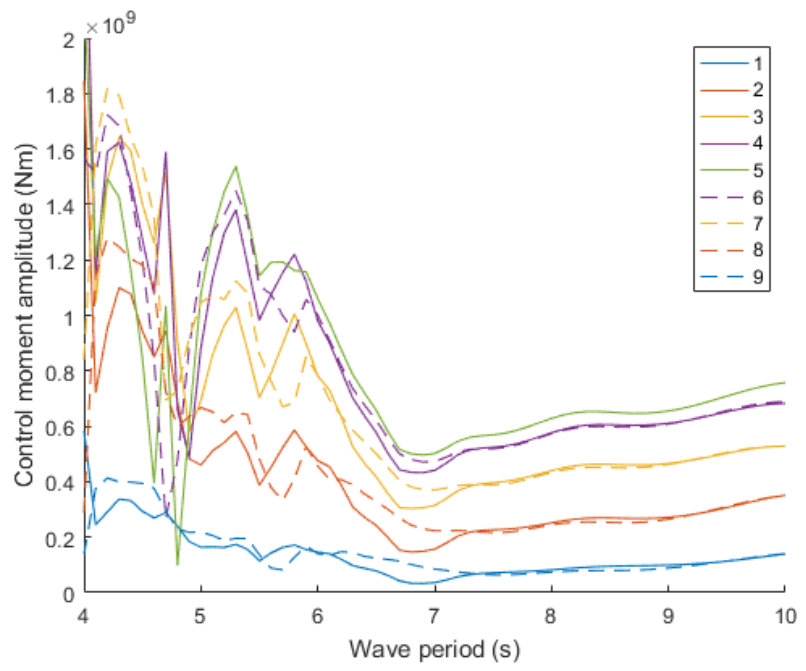

Fig. 13. The moments demanded by the control strategy for the joint roll degrees of freedom tend to be larger for joints closer to the centre of the spine, though this is somewhat dependent on wave period. Joints are numbered from fore to aft (see legend). Wave heading angle: $10^{\circ}$.

At lower wave periods, more complex behaviour emerges, and for greater wave angles, the demanded moments significantly increase at these low wave periods. This raises questions over the practical feasibility of maintaining high efficiencies for low wave periods and higher wave angles. 
Shear forces at the joints in sway (along the spine) are at least an order of magnitude lower than those in the surge and heave directions. For almost all wave angles and periods, the shear forces at the joints in the heave and surge directions are greatest at the outermost joints. Only for lower wave angles is this accompanied by lower control moments in the joints. Perhaps in these cases, the greater control forces have some causal relation with the reduction in the shear forces at the nearby joints. However, this argument does not hold for higher wave angles, where the control forces do not differ significantly along the length of the spine, yet shear forces do. Between periods of around 5 to $7 \mathrm{~s}$ and angles of 10 to $60^{\circ}$, the heave shear forces are greater at the most downstream joint than at the most upstream one (e.g. Fig. 14). This trend reverses for wave periods above about $7 \mathrm{~s}$.

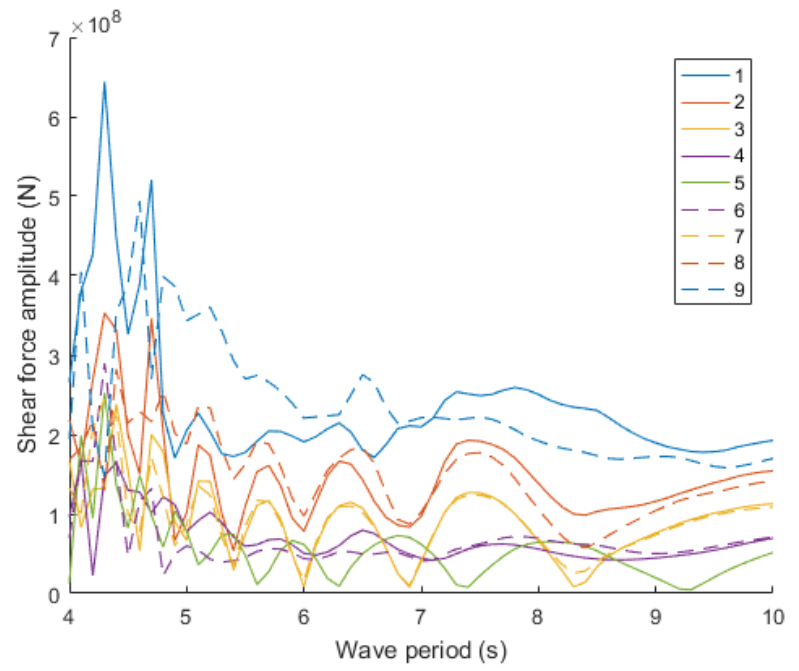

Fig. 14. Shear forces in the heave direction at each of the nine spine joints, in a wave heading of $40^{\circ}$. Joints are numbered from fore to aft (see legend).

This asymmetry in the shear forces on the joints in either half of the spine is most pronounced in wave headings of $80^{\circ}$ and $90^{\circ}$, where the heave shear forces are by far the greatest at the one or two most downstream joints (e.g. Fig. 15).

These trends bear resemblance to findings from [5]. Despite the conservative motion constraints employed here, there is also evidence of motions akin to those produced by the proposed phenomenon of a "flexural wave". The motions of the joint flexure in roll are greatest at the outermost joints for periods between 7 and 10s and angles between 10 and $50^{\circ}$ (Fig. 16 shows the effect at the central point of this range). Furthermore, the motions of the most downstream joint are greater than those of the most upstream joint.

Many of the results presented in this section offer hints of phenomena similar to those encountered in the early spine tests documented in [5], but given the complexity of the dynamics of the power-extracting spine of ducks modelled in this work, much further work is needed to clarify the exact nature of these phenomena.

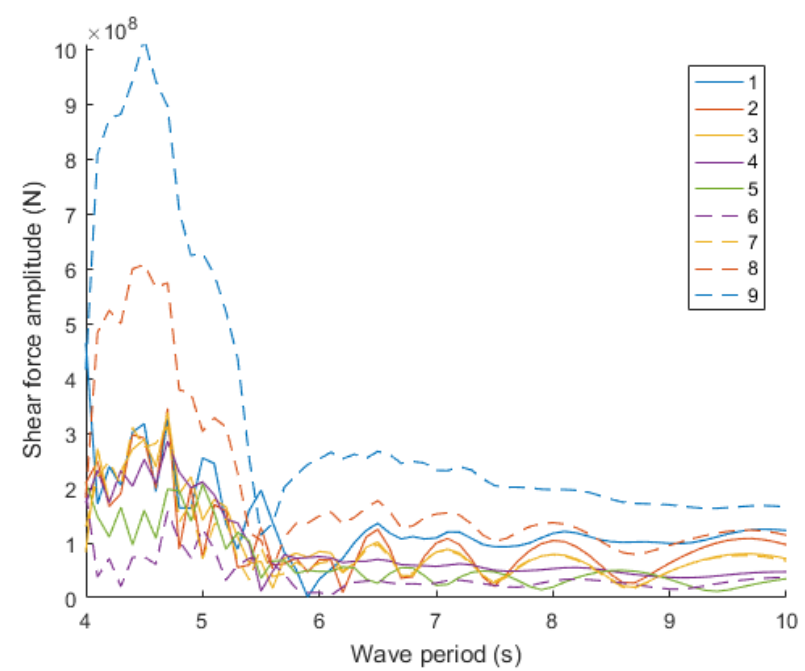

Fig. 15. Shear forces in the heave direction are greatest at the one or two most downstream joints. Wave heading angle: $80^{\circ}$. Joints are numbered from fore to aft (see legend).

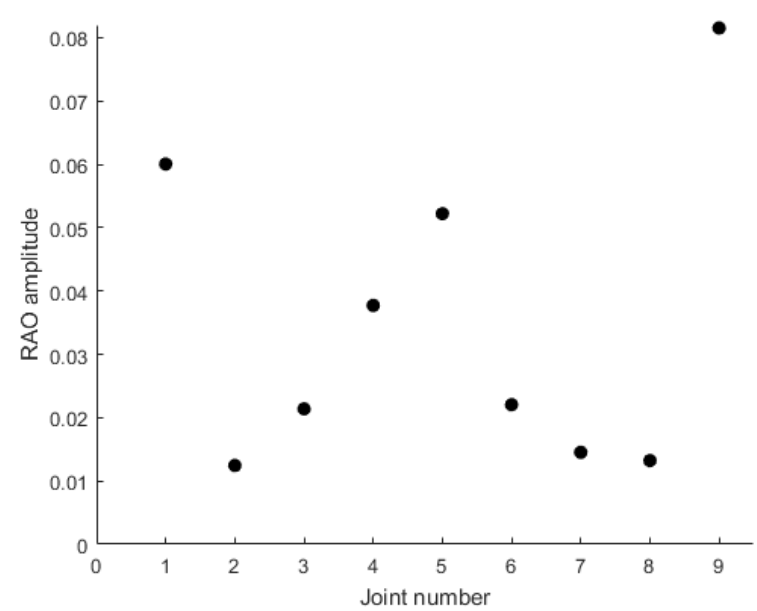

Fig. 16. Amplitudes of the motion of each of the nine joints in roll, relative to the incoming wave motion. Wave period: $8.5 \mathrm{~s}$, wave angle: $30^{\circ}$.

\section{CONCLUSIONS, LIMITATIONS AND FUTURE WORK}

In this study, an efficient frequency-domain model of a spine of ten Edinburgh duck modules has been developed. The two degrees of freedom of each spine joint, and the pitch degree of freedom of each duck module, have been linearly modelled using generalized modes. Generalized modes have also been used to analyse the shear forces acting at each spine joint. Since this is a linear model, with the hydrodynamic behaviour computed for all the degrees of freedom of the system, complex conjugate control could be applied to obtain optimal power extraction in regular waves. In order to avoid unrealistic model outputs, a weighted motion constraint has been applied to the 28 controlled degrees of freedom.

It is reassuring that many of the model outputs share similarities with the findings in [4] and [5]. It is also interesting that there are differences in the case of a full spine of ducks. The numerical model of the system developed here also allows a greater number of 
phenomena to be explored more economically. The main conclusions from the present work, regarding the design and behaviour of duck spines, are as follows:

- A significant contribution to the total power extraction should be expected from the joints, except perhaps when the wavefronts are parallel to the spine.

- Optimal power extraction may require a small angle of orientation between the spine and the incoming wavefronts.

- Optimal power extraction may require significant amounts of power being injected into certain degrees of freedom, in order to increase the extraction through others.

- The dynamics of the duck spine under the constrained optimal power take-off conditions are complex.

- Outer and inner spine joints can benefit the entire spine through their complementary dynamics and power extractions.

- Similarly, upstream ducks should have different power extraction profiles to downstream ducks.

- Shear forces acting on the joints in the sway direction (along the spine) are significantly lower than shear forces on the joints in the heave and surge directions (perpendicular to the spine).

- Surge and heave shear forces can be expected to be greater on joints at either end of the spine, and under certain conditions, highest on the downstream end joints.

- The moments on the joints, input as part of the control strategy (in yaw and roll directions), inversely correlate with the joint shear forces (in heave and surge directions), but only for conditions with small wave heading angles.

- Certain wave conditions lead to increased motions of the outermost joints, more so at the downstream end joint, even under the conservative motion constraints.

In order to extract further useful insights from this numerical model of a full spine of ducks, there are several limitations from the present work that should be addressed. Firstly, it was noted that the motion constraints most heavily affect the power extraction for the smallest wave heading angles, and so the contribution by the ducks to the total power may be significantly underestimated. Further work should aim to quantify exactly what proportion of the total power we can expect to harvest through the duck pitching alone. Since the constraint weightings were adopted from a study of a six degree of freedom system [9], the motion constraint is particularly stringent when applied to the 28 controlled degrees of freedom. Any future work that seeks to quantify the total power generating potential of the system should first quantify what constraint weightings are most appropriate. Further work should also investigate the motions of the 5 uncontrolled degrees of freedom that result through optimal control of the other 28. In order to make the model results more applicable to real sea climates, a range of wave amplitudes could also be investigated through their effects on the motion constraints. Also with regards to real sea conditions, the waterline height, centre of gravity location and mass distribution could be tuned, and the ducks should be appropriately sized for a target deployment location. The model could go beyond the analysis of regular seas by generating full time series of body responses in irregular seas, better representing a real sea climate.

A further challenge is the choice of control strategy for a practical system. A sensitivity study focussed on the control force coefficients could aim to find optimal values constrained by upper limits on the force provision. The model could also be used to analyse the amount of power exchanged in and out of each degree of freedom that does not contribute to the average power. For a given control strategy, this would give a measure of how any power take-off system inefficiencies are likely to be amplified [10], and hence the level of robustness. Finally, with regards to facilitating a fair comparison between the duck spine and other candidates for large amounts of wave power extraction, a suitable alternative metric to CWR should be devised.

\section{ACKNOWLEDGEMENT}

The authors would like to thank Professor Stephen Salter for his very constructive input to this work.

\section{REFERENCES}

[1] S. H. Salter, "Wave power", Nature, vol. 249, pp. 720-724, 1974.

[2] S. Salter, "Wave energy: Nostalgic Ramblings, future hopes and heretical suggestions", J. Ocean Eng. Mar. Energy, vol. 2, pp 399428, 2016. DOI: 10.1007/s40722-016-0057-3, [Online].

[3] S. H. Salter, "The use of gyros as a reference frame in wave energy converters", Proc. $2^{\text {nd }}$ Int. Symp. Wave Energy Utilization, Trondheim, Norway, 1982, pp. 99-115.

[4] H. E. Young and J. Pollock, "Variable coefficient control of a wave-energy device", Edinburgh Wave Power Project, University of Edinburgh, March 1985. [Online] Available: http://www.homepages.ed.ac.uk/shs/Wave\%20Energy/Old\%20 reports/

[5] S. Salter et al., "Bending moments in long spines", Edinburgh Wave Power Project, University of Edinburgh, 1984. [Online] Available:

http://www.homepages.ed.ac.uk/shs/Wave\%20Energy/Old\%20 reports/

[6] D. C. Jeffrey, D. J. E. Richmond, S. H. Salter, J. R. M. Taylor, "Second year interim report", Edinburgh Wave Power Project, University of Edinburgh, September 1976. [Online] Available: http://www.homepages.ed.ac.uk/shs/Wave \%20Energy/Old\%20 reports/

[7] D. Skyner, "Solo duck linear analysis", Edinburgh Wave Power Project, University of Edinburgh, 1987. [Online] Available: http://www.homepages.ed.ac.uk/shs/Wave\%20Energy/Old\%20 reports/

[8] D. C. Jeffrey, G. J. Keller, D. Mollison, D. J. E. Richmond, S. H. Salter, J. R. M. Taylor and I. A. Young, "Fourth year report", Edinburgh Wave Power Project, University of Edinburgh, July 1978.

[Online]

Available: 
http://www.homepages.ed.ac.uk/shs/Wave\%20Energy/Old\%20 reports/

[9] D. J. Pizer, "Maximum wave-power absorption of point absorbers under motion constraints", Appl. Ocean Res., vol. 15, pp. 227-234, 1993. DOI: 10.1016/0141-1187(93)90011-L, [Online].

[10] D. J. Pizer, "Numerical modelling of wave energy absorbers", $1994 . \quad$ [Online] Available: http://www.homepages.ed.ac.uk/shs/Wave\%20Energy/Old\%20 reports/

[11] WAMIT user manual, v7.2, WAMIT Inc. [Online]. Available: http://www.wamit.com/manual.htm

[12] J. N. Newman, "Wave effects on deformable bodies", Appl. Ocean Res., vol. 16, pp. 47-59, 1994. DOI: 10.1016/01411187(94)90013-2, [Online].

[13] D. V. Evans, "Maximum wave-power absorption under motion constraints", Appl. Ocean Res., vol. 3, no. 4, pp. 200-203, 1981. DOI: 10.1016/0141-1187(81)90063-8, [Online].

[14] J. Falnes, "Wave-Energy Absorption by Oscillating Bodies", in Ocean Waves and Oscillating Systems: Linear Interactions Including Wave-Energy Extraction. Cambridge: Cambridge University Press, 2002, ch. 6, pp. 196-224. DOI: 10.1017/CBO9780511754630, [Online].

[15] R. Yemm, D. Pizer, C. Retzler, R. Henderson, "Pelamis: experience from concept to connection", Phil. Trans. R. Soc. A, vol. 370, no. 1959, pp. 365-380, 2012. DOI: 10.1098/rsta.2011.0312, [Online]. 